\title{
Nur vergesslich oder bald dement?
}

\author{
Rund 1,6 Mio. Menschen in Deutschland sind derzeit von Demenz betroffen. Der Großteil von \\ ihnen wird vom Hausarzt betreut. Seine Aufgaben beginnen bereits bei der Frühdiagnostik.
}

? Oft fragen mich Angehörige, aber auch Patienten, ob es eine frühe Diagnostik für die Alzheimerdemenz gibt, um auch schon die "leichte Form" zu erkennen. Gibt es dazu aktuelle Empfehlungen? Ist es wirklich förderlich, bereits Frühformen zu entdecken?

In der prodromalen Phase der Alzheimerdemenz zeigen die Patienten zunehmend Gedächtnisstörungen, sie sind aber noch in der Lage, ihren Alltag allein zu bewältigen. Diese frühen Formen lassen sich nur mit spezifischen Testverfahren (z.B. subjektiv memory compliant) erfassen. Frühe positive Testergebnisse können auf ein erhöhtes prospektives Demenzrisiko hinweisen und erfordern eine regelmäßige Reevaluation. Solche Untersuchungen sollten aber nur mit Einverständnis und nach ausreichender Information des Betroffenen durchgeführt werden.

Ein unspezifisches „Screening auf Demenz" als Routineuntersuchung bei allen, d.h. auch beschwerdefreien, älteren Menschen mittels verschiedenster Kurztests ist sehr kritisch zu betrachten. Falsch-positive Diagnosen, wie sie häufig vorkommen, können Nachteile für die Betroffenen zur Folge haben. Bei Ver dacht auf eine sich anbahnende Demenzerkrankung ist grundsätzlich ein Medi-

\section{Mehr zum Thema \\ Ein kostenloses und zur Fortbildung zer- tifiziertes consilium-Themenheft „Alzhei- mer-Demenz: Frühe Diagnostik - frühe Therapie" von Prof. Pantel, können Sie bei $\rightarrow$ geriatrie@consilium.services anfordern.}

Tab. 1 Basisdiagnostik bei Demenzverdacht

\begin{tabular}{|l|l|}
\hline Untersuchungsschritte & Beispiele \\
\hline Eigen- und Fremdanamnese & \\
\hline Körperliche Untersuchung inkl. Neurostatus & \\
\hline $\begin{array}{l}\text { Krankheitsanamnese: Stoffwechselerkrankungen, } \\
\text { chronische Infektionen (Neuroborreliose, Enzephalitis), } \\
\text { Normdruckhydrocephalus, Tumoren }\end{array}$ & \\
\hline $\begin{array}{l}\text { Kognitives Assessment: Depressions-Scoring, Erfassung der } \\
\text { Alltagsfunktionen, Demenz-Detektion }\end{array}$ & $\begin{array}{l}\text { GDS, MMST, ADL, IADL, } \\
\text { DemTect, Uhrentest, CERAD }\end{array}$ \\
\hline $\begin{array}{l}\text { Laboruntersuchungen: Elektrolytstörungen, endokrine } \\
\text { Störungen, Vitaminmangel, Stoffwechselentgleisungen }\end{array}$ & $\begin{array}{l}\text { Natrium, Vit. B1, B6 und } \\
\text { B12, TSH, T3, T4, Folsäure }\end{array}$ \\
\hline $\begin{array}{l}\text { Medikamentencheck: Identifikation von Medikamenten, die } \\
\text { die Kognition beeinträchtigen }\end{array}$ & \\
\hline $\begin{array}{l}\text { Bildgebende Verfahren: zerebrale Bildgebung zum Aus- } \\
\text { schluss von Blutungen, Raumforderungen oder sonstigen } \\
\text { Veränderungen und zur Beurteilung der Atrophie-Muster }\end{array}$ & CCT, MRT \\
\hline
\end{tabular}

kamentencheck sinnvoll, um Substanzen mit potenziell schädigendem Einfluss auf die kognitive Funktion zu identifizieren (z.B. Anticholinergika, Antidepressiva, Histaminika). Weiterhin sollten auch verschiedene Laboruntersuchungen wie Elektrolytanalysen, Hormon- und Vitaminkontrollen am Anfang der Diagnostikreihe durchgeführt werden.

Eine einfühlsame und valide Frühdiagnostik der Demenz ist nicht nur erstrebenswert, um frühzeitig eine Therapie einzuleiten. Sie hilft auch dem Patienten und seinen Bezugspersonen, rechtzeitig Beratungs- und Untersuchungsangebote anzunehmen. Dies ermöglicht eine frühe selbstbestimmte Lebensplanung und schafft damit eine psychologische Entlastung.

Die erforderliche mehrstufige Diagnostik kann in der hausärztlichen Praxis mit wenig Vorbereitung umgesetzt werden. Zu jedem Zeitpunkt kann ein
Spezialist (Psychiatrie, Geriatrie, Neurologie) hinzugezogen werden.

$\rightarrow$ Prof. Dr. Hans Jürgen Heppner, MHBA Klinik f. Geriatrie HELIOS Klinikum Schwelm, Lehrstuhl Geriatrie, Univ. Witten/Herdecke, Dr.-Moeller-Str. 15, D-58332 Schwelm E-Mail: hans.heppner@uni-wh.de

\section{Haben Sie auch eine Frage an das consilium Geriatrie? \\ Haben Sie auch eine Frage, speziell aus den Bereichen Kognitive Störungen, Neurologie, Infektiologie, Schlafstörun- gen oder Dysphagie im Alter? Prof. Heppner und sein Expertenteam beant- worten diese gerne. Das ", consilium Geria- trie" ist ein gemeinsamer Service der MMW und des Unternehmens InfectoPharm. \\ Schicken Sie Ihre Frage kostenfrei an: geriatrie@consilium.services}

